\title{
COVID-19: Navigieren im Unbekannten
}

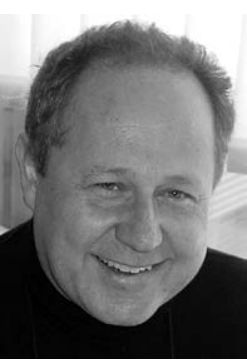

Manfred Wildner
Bibliografie

Gesundheitswesen 2021; 83: 153-155

DOI $10.1055 / \mathrm{a}-1347-8410$

ISSN $\quad 0941-3790$

(c) 2021. Thieme. All rights reserved.

Georg Thieme Verlag KG, Rüdigerstraße 14,

70469 Stuttgart, Germany

Korrespondenzadresse

Prof. Dr. med. Manfred Wildner

Pettenkofer School of Public Health

c/o Bayerisches Landesamt für Gesundheit und

Lebensmittelsicherheit

Veterinärstraße 2

85764 Oberschleißheim

Deutschland

Manfred.Wildner@Igl.bayern.de
Die Geschichte der Menschheit ist voll von Entdeckungsreisen. Sie haben teilweise mythisch-erzählerischen Charakter und reichen von den sagenhaften Reisen der griechischen Argonauten, den Irrfahrten des Odysseus oder den Abenteuern Sindbad des Seefahrers aus 1001 Nacht bis hin zu Stanley Kubricks moderner filmischer Science-Fiction-Erzählung „2001: Odyssee im Weltall“ (1968) eintausend Jahre später, zu „E.T.“ und „Star Wars“. Neben diesen Geschichten gibt es auch die tatsächliche, historisch belegte Geschichte und ihre nicht minder packenden Inhalte: Die Fahrt des Griechen Pytheas schon im vierten vorchristlichen Jahrhundert bis zum Polarkreis, die Besiedlung Grönlands durch Erik den Roten und die daran anschließende erste europäische Besiedlung Amerikas um das Jahr 1000 (Leif Eriksson), im 15. Jahrhundert die Asien- und Afrikafahrt des Chinesen Zheng He, die (Wieder-)Entdeckung Amerikas durch Christoph Kolumbus und die Indienfahrt Vasco da Gamas, im 16. Jahrhundert die Weltumsegelungen durch Magellan und Francis Drake sowie die weiteren systematischen englischen Forschungsreisen, von James Cook im 18. Jahrhundert bis zur Reise der Beagle mit Charles Darwin an Bord im 19. Jahrhundert. Das 20. Jahrhundert streckte sich dann nach den Sternen aus: 1959 mit dem ersten Weltall-Flug von Sputnik 1, 1961 mit der ersten bemannten Raumfahrt (Juri Gagarin), 1969 glückte mit Apollo 11 die Landung auf dem Mond, 1997 die Landung eines Rovers auf dem Mars, 2014 eine Landung auf dem Kometen 67P/Tschurjumow-Gerassimenko (ESA-Mission Rosetta).

Möglich wurden diese Fahrten nicht nur durch Wagemut und Geschick, sondern im hohen Maß auch durch Technologie und Wissenschaft - v. a. auf dem Gebiet der Navigation, der Steuermannskunst (Latein: Navigare/Sanskrit: Navgathi). Die Instrumente dafür wurden in geschichtlich langer Reihenfolge entwickelt: Lot und Kompass, Astrolab und Jakobsstab, Sextant und Chronometer, Astronavigation und Funkpeilung und mit dem Fortschreiten von
Raumfahrt und digitalen Technologien die heutigen Systeme der globalen Navigationssatelliten wie das amerikanische GPS, das russische GLONASS, das chinesische Beidou und das europäische Galileo.

Der Bezug zum Gesundheitswesen? Hier sei eine Analogie zur COVID-19-Pandemie 2020/21 versucht. Die Notwendigkeit zur möglichst genauen Positionsbestimmung anhand der Meldedaten und Studienergebnisse, die Festlegung eines bestmöglichen gesellschaftlichen Kurses in den Konferenzen der politischen Staatenlenker und das Halten und beständige Nachjustieren eines solchen Kurses ähnelt dem Vortasten ins Unbekannte der großen tatsächlichen Erkundungsfahrten und auch der sagenhaften Durchfahrt zwischen „Skylla und Charybdis“, als Metapher einer Balance zwischen einem Zu-Wenig und einem Zu-Viel, in der Hoffnung auf ein glückliches Ende der (Irr-)Fahrt. Damals wie heute kommt der Navigation als dafür tragender Wissenschaft und Kunst eine zentrale Bedeutung zu.

Was wären die zentralen „Navigationsinstrumente“, um im Bild zu bleiben, während einer Pandemie? Um bei der Positionsbestimmung als Ausgangspunkt zu beginnen: Im Vordergrund stehen hier die Meldefälle nach dem Infektionsschutzgesetz (IfSG), überwiegend auf Basis der pflichtigen Labormeldungen und ergänzt um weitere Angaben wie Infektionsumfeld, Erkrankungsbeginn und Vitalstatus (cf. www.rki.de). Auch wenn man sich hier in Analogie zur Wasserstandsmeldung unter dem Kiel größtmögliche zeitliche Nähe wünscht - dieser Wunsch wird bezogen auf den tatsächlichen Zeitpunkt der Erregerübertragung wohl auf absehbare Zeit eine Utopie bleiben, trotz technischer Neuerungen wie der DEMIS-Plattform zur raschen elektronischen Übermittlung der Labordaten. Nicht nur, dass auch schnelle Meldungen hinsichtlich ihrer Richtigkeit vor einer weiteren Übermittlung zu prüfen sind und hier ein Trade-off zwischen Geschwindigkeit und Genauigkeit zu berück- 
sichtigen ist. Mehr noch ist zu bedenken, dass diese Meldezahlen der Laboruntersuchungen immer zu spät kommen und nur die Vergangenheit abbilden können, gleichsam einen „Blick in den Rückspiegel“ geben. Der eigentlich interessierende Moment „unter dem Kiel“, der Zeitpunkt der Infektionsweitergabe, ist dann schon längst Vergangenheit. Er wird dabei gefolgt von einer Latenz- und Inkubationszeit bis zu den ersten wahrnehmbaren Krankheitssymptomen, welche zwischen 2 Tagen und 2 Wochen liegen kann, im Mittel bei 5-6 Tagen. Kritisch ist, dass sich schon 2-3 Tage vor dem Symptombeginn die Hälfte der Infektionsweitergaben durch die betroffene Person ereignet hat. Rechnet man dann noch die Zeiten bis zur Durchführung eines Abstriches für eine laboranalytische Untersuchung, die Laborlaufzeiten und die Zeiten der Meldewege hinzu, sind bereits 10 Tage vergangen, wenn die Meldung in den Landes- und Bundesstatistiken erscheint, dort bewertet und zur Grundlage von Maßnahmen genommen wird. Und analog werden sich die Effekte von rasch eingeleiteten Gegenmaßnahmen wiederum erst 10 Tage später erstmals an den Meldefällen erkennen lassen [1].

Gleichzeitig ist noch ein zusätzlich wirksamer Trend im Auge zu behalten, gleichsam eine langsame Abdrift dieser Meldezahlen, welche sich aus der relativen „Ebbe“ und „Flut“ der insgesamt durchgeführten Testzahlen bei der Suche nach Infektionen ergibt. Angesichts eines „Dunkelfeldes“ zwischen einem und fünf unerkannt gebliebenen Infektionen je tatsächlichem Meldefall ist auch nicht verwunderlich, dass bei einer Ausweitung der Testungen auch mehr Fälle gefunden werden. Dies allerdings in unbeständiger Proportionalität von Hellfeld und Dunkelfeld, was die mittel- und langfristige Interpretation von absoluten Werten und Trends erschwert [2]. Hinzu kommen Unvollkommenheiten der Testsysteme, welche falsch-positive und falsch-negative Testergebnisse bedingen und bei der Interpretation der Meldezahlen ebenfalls mit ins Kalkül gezogen werden müssen, insbesondere in Niedrigprävalenzlagen [3-5].

So hält man sehnlich nach festen Bezugspunkten Ausschau, nach Leuchttürmen und markanten Markierungen. Solche Fixpunkte mit gegenüber den Meldefällen verbesserter Validität existieren tatsächlich. Es handelt sich hier zum einen um die gemeldeten Todesfälle an bzw. mit COVID-19, wobei diese genaue Unterscheidung für die Aufgabe der Surveillance und Trendbestimmung von untergeordneter Bedeutung ist. Der Anteil von COVID-19 Diagnosen ohne kausalen Bezug wird dabei auf etwa $12 \%$ geschätzt [6]. Die Todesfälle treten im Mittel etwa 2-3 Wochen nach der Infektion ein, mithin etwa eine Woche nach Eingang der Meldungen. Der Vorteil ist, dass die Todesfälle vermutlich annähernd vollständig erfasst werden und bei Kenntnis der anzunehmenden Sterblichkeit unter den Infizierten einen Rückschluss auf die tatsächlichen Infektionszahlen zulassen. Auch hier ist für eine relative Trendbewertung die exakte Bestimmung des Sterberisikos bei Erkrankung (Letalität bzw. englischsprachig Case Fatality Rate, CFR) bzw. des Sterberisikos bei Infektion (Infection Fatality Rate, IFR) zunächst nicht erforderlich. Diese relative Trendbewertung sollte allerdings nur anhand der absoluten Fallzahlen oder aber einer spezifischen bevölkerungsbezogenen Mortalität erfolgen, nicht anhand der vergleichsweise volatilen Meldefall-bezogenen Sterblichkeit. Eine europaweite Übersicht zum bevölkerungsweiten (Excess-)Sterblichkeitsgeschehen bietet das europäische Projekt EUROMOMO (www.euromomo. eu). Der Vollständigkeit halber seien auch noch die Todesfälle wegen COVID-19 erwähnt - die namenlosen und ungezählt gebliebenen tragischen Schicksale derjenigen, welche wegen einer Priorisierung des COVID-19-Geschehens bei Diagnose, Therapie und Rehabilitation eine Posteriorisierung ihrer medizinischen Versorgung erfahren und im Weiteren ihr Leben verloren haben - oder auch in Folge der ökonomischen Auswirkungen insbesondere in ärmeren Weltregionen.

Einen weiteren Orientierungspunkt bieten auch die Belegungszahlen der Intensivpflegebetten durch Patienten/innen mit COVID19-Nachweis, welche einen Höhepunkt etwa drei Wochen nach der unbemerkt gebliebenen Infektion aufweist, mithin etwa 10-12 Tage nach einem Gipfel bei den Meldezahlen. Diese lassen sich ebenfalls im zeitlichen Trend und im regionalen Vergleich beobachten und analysieren (www.divi.de). Nicht zuletzt bildet auch die zeitvariable Reproduktionszahl $R(t)$ einen Trend vergleichsweise robust ab: Da sich $\mathrm{R}(\mathrm{t})$ auf das Verhältnis der um die Wochenperiodizität geglätteten Meldezahlen bezieht, lässt sich dieses Verhältnis z. B. auch stabil aus der zeitveränderlichen kumulativen 7-Tages-Inzidenz für jeweils ein 4-tägiges serielles Intervall abschätzen. Gegenüber den langfristigen Trends einer Veränderung des Verhältnisses von Hell- und Dunkelfeld bleibt diese Maßzahl damit anders als die Anzahl der Meldefälle selbst - auch im zeitlichen Längsschnitt unempfindlich.

Zudem lassen bei einer längsschnittlichen Analyse die Meldefallzahlen als altersspezifische Inzidenzen über einen begrenzten Zeitraum durchaus relevante Interpretationen im Vergleich der Altersgruppen untereinander zu: Sie lassen z. B. Ende des Jahres 2020 zunächst einen Anstieg bei den jungen, berufstätigen Erwachsenen erkennen, welchem erst zeitlich anschließend ein Anstieg bei den Kindern und zuletzt ein Anstieg bei den hochaltrigen, über 80jährigen Bevölkerungsgruppen folgt, der dann eine Eigendynamik entwickelt. Es liegt nahe, in dieser zeitlichen Abfolge bei den Meldezahlen auch eine zeitliche und womöglich kausale Abfolge des Infektionseintrags zu vermuten. Die überschießende Dynamik bei den hochaltrigen Altersgruppen könnte einer institutionellen Unterbringung in Alten- und Pflegeheimen und einer dort besonders intensiven Nachverfolgung von Kontaktketten geschuldet sein. Wesentlich sind auch regionale Analysen - kleinräumig als small area Analysen, Analysen auf Ebene der Kreise, der Länder, der Nationalstaaten und darüber hinaus. Wichtig ist, neben der Beobachtung innerhalb der gegebenen regionalen Zuständigkeiten auch immer den weiteren Horizont im Auge zu behalten - ein erhöhter Infektionsdruck z. B. in Folge von Mutationen und damit entstandenen „Variants-of-Concern“ ( $\mathrm{VoC})$ machen ebenso wie Eisberge nicht an Verwaltungs- oder Staatsgrenzen halt.

Ergänzen lassen sich solche „Augapfelnavigationen“ auch noch durch eine Betrachtung des zeitlichen Verlaufs der Testpositivrate der durchgeführten Testungen als Maß für den augenblicklichen Infektionsdruck, wobei „Verdünnungs“- und „Konzentrations“-Effekte durch die Zu- und Abnahme der Gesamtzahl der durchgeführten Testungen mit zu beachten sind. Dass über die Analyse dieser Routinedaten hinaus auch noch gezielte Studien wertvolle Beiträge liefern können, z. B. als repräsentative Erhebungen mit validierter Methodik, als seroepidemiologische Studien, als stichprobenartige Gesamtgenomsequenzierung, als Sentinel-Erhebungen, als Umweltbeprobung oder als Erhebungen zu Impfraten und Impfeffektivität, ist an dieser Stelle ebenfalls deutlich zu sagen. Dies 
beinhaltet auch eine aufmerksame Beobachtung der nationalen und internationalen wissenschaftlichen Fachveröffentlichungen.

An eine solche sachkundige Positionsbestimmung, welche über „die Zahlen“ der Tagesmedien weit hinausgeht, kann sich wiederum eine intelligent und sachkundig beratene Festlegung des bestmöglichen Kurses für die Zukunft anschließen. Dafür passende Instrumente sind u. a. Hearings, Modellierungen und formale Methoden der Konsensfindung unter Experten. Dass Politik durch Evidenz nur informiert werden kann und politische Entscheidungen zu Pandemiefragen nicht allein aus den Domänen Epidemiologie, Virologie und Infektionsschutz informiert werden dürfen und können, ist ebenfalls festzuhalten. Entscheidungen müssen zudem häufig auch unter Unsicherheit getroffen werden. Das Halten und Nachjustieren eines gewählten Kurses ist wiederum vor allem eine Kunst - die politische „Kunst des Möglichen“ und verlangt gelingende und intensive Risiko- und Krisenkommunikation [7-9]. Die zuletzt genannten Aufgaben der Kurswahl und des Kurshaltens oder auch Nachjustierens sind allerdings Aufgaben der Kapitäne, nicht mehr der Navigationsoffiziere.

Die Beiträge in diesem Heft wollen in diesem Sinn ihren Beitrag zu einer evidenzinformierten, bestmöglichen Entscheidungsfindung leisten: Zu Veränderungen im Sportangebot durch die COVID19 Pandemie, zu Auswirkungen der Ausgangsbeschränkungen im Zusammenhang mit Covid-19 auf Supermarktbesuche, zur Sinnhaftigkeit der Desinfektion öffentlicher Flächen zur Prävention von SARS-CoV-2, zur Rolle von Rapid Reviews in Zeiten von COVID-19 - Erfahrungen des Kompetenznetzes Public Health, zur Akzeptanz von Versorgungsangeboten zur ausschließlichen Fernbehandlung am Beispiel des telemedizinischen Modellprojekts „docdirekt“, zur Entwicklung einer Schulung zur Förderung der Gesundheitskompetenz von Patienten mit variablem Immundefekt (CVID), zur Beziehung zwischen sozialer Mediennutzung, subjektiver Gesundheit und Risikoverhalten im Kindes- und Jugendalter, zu Determinanten der kognitiven Entwicklung bei Kindern, zur Evaluation der Sprachaufgaben des sozialpädiatrischen Entwicklungsscreenings für Schuleingangsuntersuchungen (SOPESS), zum Zusammenhang zwischen sozioökonomischem Status und Entwicklungsstand sowie mit einer Stellungnahme des „Expertenkreises Aerosole“: Aerosole und SARS-CoV-2 - Entstehung, Infektiosität, Ausbreitung und Minderung luftgetragener, virenhaltiger Teilchen in der Atemluft.

Um am Ende noch einmal zum Anfang zurückzukommen: Das Ziel solchen Navigierens ist in der weiteren Zielsetzung wie auch in der Vergangenheit paradoxerweise nicht so sehr das ursprünglich vielleicht gesuchte Abenteuer, sondern im Gegenteil eine sichere Fahrt auch durch gefährliche Gewässer. Dafür ist eine solide theoretische wie auch praktische Aus-, Weiter- und Fortbildung der betroffenen Berufsgruppen ebenso notwendig wie das sorgfältige proaktive und prospektive wissenschaftliche Kartieren der zu befahrenden Regionen, wie dies bspw. in den Seekarten und den auch finanziell kostspieligen Satellitennavigationssystemen zum Ausdruck gekommen ist und in Primärdatenstudien, Sekundärdatenanalysen und Modellierungen, Gesundheitsberichte und auch Konzepte zur pandemic preparedness, zu Leit- und Richtlinien über- setzt werden könnte. Notwendige Stützpunkte auf diesen Fahrten könnten die entsprechend auf- und ausgerüsteten Gesundheitsämter werden, die für diese theoretische und praktische Ausrüstung notwendigen Navigationsschulen die zugehörigen universitären bzw. akademischen Strukturen: ÖGD-Lehrstühle und Schools of Public Health. Die Investition von Mitteln in solche Vorhaben dürfte sich ebenso wie bei der Seefahrt durch die zu erwartenden Erträge mehr als lohnen - neue Abenteuer in einer Welt der Ideen inbegriffen, die von Sputnik V bis „One Health“ und vermutlich auch darüber hinaus reichen.

\section{Interessenkonflikt}

Die Autorinnen/Autoren geben an, dass kein Interessenkonflikt besteht.

\section{Literatur}

[1] Küchenhoff H, Günther F, Höhle M et al. Analysis of the early Covid-19 epidemic curve in Germany by regression models with change points. Preprint www.medrxiv.org. doi: https://doi.org/10.1101/2020.10.29. 20222265

[2] Neuhauser H, Thamm R, Buttmann-Schweiger $\mathrm{N}$ et al. Ergebnisse seroepidemiologischer Studien zu SARS-CoV-2 in Stichproben der Allgemeinbevölkerung und bei Blutspenderinnen und Blutspendern in Deutschland (Stand 3.12.2020). Epid Bull 2020; 50: 3-6

[3] Guenther F, Bender A, Höhle M et al. Analysis of the COVID-19 pandemic in Bavaria: adjusting for misclassification. Preprint www.medrxiv.org. doi: https://doi.org/10.1101/2020.09.29.20203877

[4] Kucirka LM, Lauer SA, Laeyendecker O et al. Variation in False-Negative Rate of Reverse Transcriptase Polymerase Chain Reaction-Based SARS-CoV-2 Tests by Time Since Exposure. Ann Intern Med 2020; 173 : 262-267

[5] Bergmann H, Engelhardt D, Storfinger N. Kennzahlen rund um die Corona-Pandemie. eine Betrachtung aus Sicht der Statistik. Fürth. 2020; URL https://www.statistischebibliothek.de/mir/receive/ BYMonografie_mods_00001532, access 08.02.2021

[6] Bayerisches Landesamt für Gesundheit und Lebensmittelsicherheit. Übersicht der Fallzahlen von Coronavirusinfektionen in Bayern. URL https://www.lgl.bayern.de/gesundheit/infektionsschutz/ infektionskrankheiten_a_z/coronavirus/karte_coronavirus/index.htm, access 07.02.2021

[7] Wildner M. Präventionsberichterstattung: Evidenz - und was noch? In: Robert Koch-Institut, Bayerisches Landesamt für Gesundheit und Lebensmittelsicherheit Hrsg. Über Prävention berichten - aber wie? Methodenprobleme der Präventionsberichterstattung. Beiträge zur Gesundheitsberichterstattung des Bundes. Berlin: RKI; 2020: 133-138

[8] Von Philipsborn P, Garlichs D, Wildner M et al. Politische Umsetzung von Verhältnisprävention auf Bevölkerungsebene: Herausforderungen und Erfolgsfaktoren. Gesundheitswesen 2020; 82: 386-388

[9] Wildner M. Evidence-based Public Health (EBPH) - Gesundheitspolitische Beratung und Information der Öffentlichkeit. J Health Monitoring 2020; 5: 17-18 